\title{
La mujer profesional de la educación \\ en la vida nacional peruana
}

\author{
Female professional educators \\ in the Peruvian life
}

Elsa Bustamante Quiroz ${ }^{1}$

\begin{abstract}
RESUMEN
En este artículo se expone un análisis de la participación de la mujer profesional de la educación en la vida nacional peruana entre las últimas décadas del siglo pasado y las primeras del presente, a partir de la experiencia y la visión de tres mujeres representativas. La investigación se ha realizado desde un enfoque biográfico y se ha utilizado la entrevista semiestructurada. Se concluye que, si bien hay presencia femenina relevante, como colectivo la mujer se ha desempeñado fundamentalmente en la docencia mas no en la conducción política social. La trayectoria de quienes han logrado tener un papel trascendental, debe motivar a potenciar capacidades y circunstancias que permitan un rol cada vez más significativo.
\end{abstract}

\section{Palabras clave}

participación de la mujer, docente mujer, formación del docente

\begin{abstract}
This article presents an analysis of the participation of female professional educators in Peruvian life in the past century's last decades and the first decades of the current century through the experience and vision of three female representatives. This research was done using a biographical approach and a semi-structured interview. In conclusion, although the female presence has been relevant, women as a group have primarily developed in the teaching field but not in social politics. The pathways of those who have achieved important roles should motivate the enhancement of capabilities and conditions for women to have more significant roles.
\end{abstract}

\section{Keywords}

Women's participation, female teacher, teachers training

\section{INTRODUCCIÓN}

En el Perú, la feminización del magisterio puede dar la imagen de una participación preponderante de la mujer en el campo de la educación, sin embargo, esta situación oculta un ejercicio relegado. Como señala Valdés (2000), la acción de las mujeres ha estado enmarcada en una estructura patriarcal, que permanece aún en Latinoamérica, limitándolas constantemente al ámbito de lo doméstico y haciéndolas invisibles en la acción pública. Así, entre 1960 y 2019, que es el período en el que se centra esta investigación, ha habido 50 Ministros de Educación en el Perú (Ministerio de Educación [MINEDU], 2019) de los cuales solo cinco han sido mujeres: Mercedes Cabanillas Bustamante,

Doctora por la Universidad de Salamanca, Especialista Universitario en Intervención Clínica en Lectura y Escritura por la Universidad Complutense de Madrid, Licenciada en Educación Especial por la Universidad Femenina del Sagrado Corazón (UNIFE).

Docente Ordinario en la Universidad Marcelino Champagnat y en la UNIFE. Directora del Instituto de Investigación de la Universidad Marcelino Champagnat. Experiencia en docencia en educación básica y superior (pregrado y postgrado), en investigación educativa y en diseño y ejecución de proyectos educativos. 
Gloria Helfer Palacios, Patricia Salas O’Brien, Marilú Martens Cortés y Flor Pablo Medina.

Otro dato que hay que tener en cuenta es la poca participación de la mujer pedagoga en el campo de la investigación en educación. En el Registro de investigadores en Ciencia y Tecnología del Sistema Nacional de Ciencia, Tecnología e Innovación Tecnológica (SINACYT) figuran 127 investigadores calificados en la sub área de Ciencias de la Educación, de ellos solo 40 son mujeres Sin embargo, otro apunte importante que aportan las estadísticas de CONCYTEC es que de estos investigadores solo 50 son profesionales de la Educación (Consejo Nacional de Ciencia, Tecnología e Innovación Tecnológica [CONCYTEC], 2019). Al parecer, en este campo las limitaciones estan más ligadas a la profesión que al género, así, por ejemplo, en la Sociedad de Investigación Educativa Peruana (2019), si bien el número de varones es mayor (51) al de mujeres (46), no hay una brecha importante, más bien, en el grupo total solo se encuentran ocho profesionales de la educación, de los cuales dos son mujeres.

No obstante, las limitaciones impuestas no han podido opacar la trascendencia de la mujer peruana en el campo de la educación. El objetivo de este artículo es presentar un análisis de la relevancia que han tenido las mujeres educadoras en la vida social peruana entre las últimas décadas del siglo pasado y las primeras del presente, a partir de la experiencia y de la visión de tres mujeres representativas: Gloria Helfer Palacios, Nelly Villalobos Bustamante y Socorro del Pilar Cardó Franco

\section{Método}

Este estudio se ha realizado desde un enfoque biográfico. Se recogió datos a través de entrevistas semiestructuradas, las que fueron grabadas con la autorización de las participantes, luego se procedió a convertir los audios a material textual para proceder a su división en unidades de texto que fueron organizadas según dos ámbitos de participación: el político social y el de la docencia, distinguiendo en cada uno las siguientes categorías (figura 1 ):

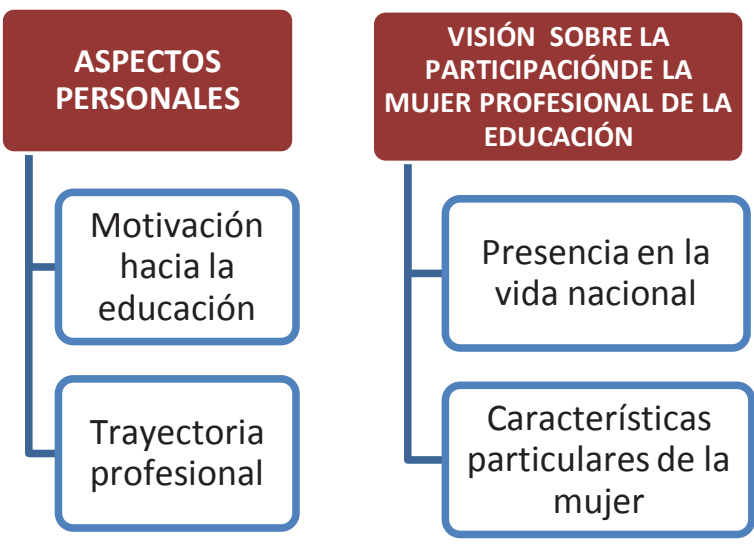

Figura 1. Categorías y subcategorías para el análisis e interpretación de datos

Los resultados fueron presentados a las participantes quienes manifestaron su conformidad con la interpretación realizada (validación comunicativa).

\section{1. ÁMBITO POLÍTICO SOCIAL}

Se analizará en este punto la acción de la mujer en organizaciones del Estado y de la sociedad civil enfocadas en la educación, así como su presencia en cargos de gobierno.

La primera vez que una mujer juramentó el cargo de Ministra de Educación fue el 29 de junio de 1987, durante el gobierno de Alan García; era, además, la primera vez que una mujer asumía la dirección de un Ministerio en el Estado peruano, aunque no fue solo en el campo de Educación, ya que, a la vez que Mercedes Cabanilllas asumía la cartera de Educación, Ilda Urizar hacía lo propio en Salud. Si bien esto se presenta como reconocimiento tardío a la capacidad femenina luego de 166 años vida republicana, hay que recordar que solo en 1955 se otorgó ciudadanía a las mujeres peruanas mayores de edad que supieran leer y escribir, y que su participación en elecciones se inició el 7 de setiembre de 1956.

Mercedes Cabanillas Bustamante al juramentar como Ministra tenía ya una amplia trayectoria en el devenir político del país; era, y es, una figura emblemática del entonces partido gobernante, el Partido Aprista Peruano, al cual ha pertenecido desde los 14 años. En una entrevista dada al periódico La República señala que prueba de su joven ingreso al partido es que el día que cumplió 15 años llenó su casa paterna de amigos de la 
Juventud Aprista (Núñez, 2013, párr. 1). Fue Ministra de Educación de junio de 1987 a agosto de 1998 y de mayo a julio de 1990. Cuando Cabanillas dejó el cargo la sucedió otra mujer: Gloria Helfer.

Gloria Helfer Palacios nació en Lambayeque $y$, como muchas personas que han optado por la docencia, se sintió inclinada a la enseñanza desde la niñez. La vocación temprana no es algo extraño en la vida de las educadoras de trayectoria trascendente. Ella relata "A los 14 años yo ya daba clases a mis compañeritas de curso. Nunca dudé que cuando tuviera que escoger una profesión, iba a escoger Educación. Hacía otras cosas, hacía teatro, hacía filosofía, pero, era maestra". Estudió Educación en la Pontificia Universidad Católica del Perú y cuando viajó a Lovaina (Bélgica) a estudiar Sociología lo hizo para entender mejor la educación, no para ser socióloga. De regreso al Perú ingresó a trabajar en el Ministerio de Educación (1972-1978), en la época de la Reforma de la Educación; época marcada por retos, innovaciones, imprecisiones y entusiasmo. Época de "gran reflexión pedagógica" según Helfer, en la que, encabezados por Salazar Bondy, fueron emergiendo en el escenario educativo nacional figuras como Walter Peñaloza, César Picón, Shona García, Carmen Montero, entre otros pedagogos.

Gloria Helfer ha sido profesora en el nivel superior en el Pedagógico de Ayacucho y en la universidad de Ayacucho; esto le valió conocer, junto con sus alumnas practicantes, la realidad de la educación rural peruana en los años 70. Pero, también, ha conocido lo que es ser profesora de aula de educación básica en una institución educativa del Estado. Según relata, fue de su aula del colegio Jorge Chávez, de Santiago de Surco, de donde salió para ejercer como Ministra de Educación durante el primer gobierno de Alberto Fujimori y allí regresó cuando terminó su corto paso por el gabinete. En esta institución fue dirigente "muy humilde" del Sindicato Unitario de Trabajadores en la Educación del Perú (SUTEP). Convencida siempre de que no se puede ser buen político ni buen dirigente si no se es buen maestro, buscó enseñar la Historia de una manera dinámica, innovadora, con historietas y laboratorio al que los alumnos acudían a recibir las clases. Recordando esa época, refiere:
Estábamos uniendo la política, lo sindical y lo pedagógico. Inclusive, escribimos en esa época un librito, con Lucha, con Manuel, escribimos un librito al que le llamamos "Educando también estamos luchando", que era el reverso de "Luchando también estamos educando", que era el lema del SUTEP; y nosotros, sin ser antisindicalistas, ni mucho menos, volteamos el lema y decimos "Educando también estamos luchando", es decir, si tú educas, ya estas trasformando el país, ya estás trasformando la humanidad, porque ese es nuestro compromiso como educadores.

Para ella el período en el Ministerio fue una época marcada por el trabajo en equipo. Cuando llegó convocó a personas comprometidas con la educación del país conformando lo que se conoció como el Grupo de los Cien, germen de Foro Educativo, "un colectivo muy amplio, donde mucha gente participa y que entra con un gran entusiasmo por hacer una gran transformación en educación", teniendo como ejes claves: una propuesta pedagógica a largo y mediano plazo, encaminada hacia el Consejo Nacional de Educación; la lucha contra la corrupción; y el programa de salud y alimentación escolar La escuela defiende la vida. Durante los pocos meses que estuvo en el cargo (del 28 de julio al 12 de diciembre de 1990) puso en marcha este programa que proporcionó alimentación a escolares en un período extremadamente crítico para el país acerca del cual Morillo (2002) comenta: "El shock del 8 de agosto de 1990 provocó una inflación mensual de 392\%, con una caída brutal de la producción en el segundo semestre de ese año $(-20 \%)$, colocó a la mayoría de la población en situación de pobreza absoluta" (p. 4). Fueron tres millones doscientas mil raciones las que se entregaron, en muy poco tiempo, gracias a una red de personas que ya estaban trabajando a nivel nacional, fundamentalmente grupos vinculados a la Iglesia y a las Organizaciones no Gubernamentales (ONG). Refiriéndose a este logro, Gloria Helfer afirma: "no hay nada muy individual que digamos: eso no lo hace una persona, eso se hace cuando hay un grupo humano que está involucrado". 
Luego de su paso por el Ministerio, permaneció en la vida política como congresista y fue presidenta de la Comisión de Educación del Congreso en la época en la que se elaboró la Ley General de Educación, la cual, según señala, es fruto de un trabajo colectivo muy interesante que tenía como antecedente la consulta nacional dirigida por el padre Ricardo Morales: La educación, puertas abiertas a la vida, iniciada cuando asumió la presidencia Valentín Paniagua. En la Ley, vigente hasta hoy, ya se hace referencia al Consejo Nacional de Educación, que se crea para propiciar la participación de la sociedad en diálogo con el Estado, teniendo en vista el Proyecto Educativo Nacional. En cuanto a producción, su mayor orgullo es haber puesto al alcance de los niños esta Ley y la sustentación jurídica del derecho a la educación, a través de folletos de difusión, amenos y atractivos, que llevó por todo el país.

A la par del trabajo político, sindical, social y profesional, ella ha desempeñado el rol de madre y esposa. Tiene un matrimonio de casi 50 años y dos hijas. ¿Cómo lo ha hecho? Según señala siempre hay un costo para la familia, pero si lo ha podido lograr es porque su familia ha estado involucrada, siendo su esposo su principal apoyo y empuje en el momento de asumir responsabilidades y cumplir con las funciones que se le encargaban:

Era el primero que me decía "Acepta, tú puedes hacerlo", "No, pero mira, si yo soy una profesorita, como me van a meter.... Yo soy maestra, que no me metan a mi" y él me decía "Claro que puedes" y ha sido siempre mi apoyo maravilloso.

Lo conocí en Lovaina y ha sido mi apoyo incondicional, y mis hijas también, muy involucradas siempre en política, muy dinámicas. Entonces, esa ha sido la ventaja, que no es el enfrentamiento con lo que la madre hace, sino que cobra sentido lo que la madre está haciendo. Por supuesto era llegar a contar todo, a compartir. Por eso hablábamos de calidad de tiempo, pero igualito, la ausencia es la ausencia, y tiene costos, y tenemos que saber que no existe la fórmula mágica.

Según Helfer, los factores culturales que han exigido a la mujer atender muchas cosas a la vez (cocinar, atender al bebé, mientras consuela al otro hijo que llora, por ejemplo) han hecho que sea capaz de tener una diversidad de miradas, que pueda tener una visión panorámica de una situación, visión que hace mucha falta en política y en otros campos en el Perú. Por eso ella dice que si se quisiera concretar en una palabra su labor en la educación peruana, esta sería juntar: juntar a personas que están involucradas con la educación en una experiencia pedagógica, en un movimiento, en el Grupo de los Cien, en el equipo de gobierno, en instituciones como Foro Educativo, juntar instituciones para vencer al cólera:

Toda la vida he tratado de juntar, ¿por qué?, porque somos un país terriblemente dividido, culturalmente, enfrentado. Mira, la política es un lugar de enfrentamiento continuo, de agresión, entonces el poder juntar es la única opción que tenemos de crear cosas,

Por lo diversas que somos (las mujeres), por lo polifacéticas que somos, entonces también podemos juntar lo diferente y yo creo que el Perú necesita mucho de esa actitud de reunir, de juntar, de potenciar, de hacer sinergia.

La tercera Ministra de Educación que ha tenido el Perú es la socióloga Emma Patricia Salas O'Brien. Salas fue ministra entre el 28 de julio de 2011 y el 31 de octubre de 2013. Marilú Martens Cortés fue Ministra ente diciembre de 2016 y setiembre de 2017, y Flor Aidee Pablo Medina ejerce el cargo desde marzo de 2019.

La participación de la mujer en el ámbito político social, no se ha limitado al área de la administración estatal, sino que ha aportado significativamente también, y quizá con mayor impacto, desde la sociedad civil. Así, se aprecia que en instituciones que representan una $\mathrm{vOz}$ autorizada en el campo de la educación, como 
es el caso de Tarea, Fe y Alegría, Instituto de Investigación José Antonio Encinas e Instituto de Estudios Peruanos, su labor ha sido predominante, tanto en su formación como en su consolidación.

\section{2. ÁMBITO DE LA DOCENCIA}

En este punto se hará una descripción y análisis del papel de la mujer como docente dedicada al quehacer diario en el contexto escolar; es decir, la educadora protagonista de la práctica educativa.

En el campo del ejercicio docente en la educación escolar se puede apreciar la presencia imponente de la mujer en el período en el que se centra esta investigación. Mientras que carreras consideradas masculinas en el siglo pasado poco a poco fueron recibiendo mayor afluencia de mujeres hasta casi equiparar el número según sexo, las carreras femeninas se han mantenido con esta característica, como es el caso de enfermería y educación. Esta preponderancia se aprecia no solo en el marco del aula, sino, también, en la dirección y promoción de instituciones educativas de marcada proyección.

Uno de los gobiernos que más ha trabajado por la educación en el Perú ha sido el del general Manuel Odría; simpatizantes o detractores lo reconocen como un Presidente preocupado por la educación, en especial por la de los grupos vulnerables, entre ellos las mujeres. Al respecto afirma Chávez (2006) que el gobierno de Odría "Tuvo una gran significación para el ámbito de lo educativo: demostró que, material y económicamente, el Estado puede responder a las exigencias populares" (p. 56). Entre sus obras más significativas está la creación de las Grandes Unidades Escolares (GUE); en una de ellas, la GUE Mercedes Cabello confluyeron las trayectorias pedagógicas de tres grandes educadoras: María Ventocilla Cámara, Blanca Dávila Recio y Rita Castro Ramos. Mujeres como ellas lograron dar a la educación peruana de la segunda mitad del siglo XX el empuje que requería para sobrepasar la barrera de la marginación de género. Si bien es cierto, aún la mujer no puede acceder a los mismos beneficios que el varón, la brecha entre ellos, en lo relacionado al acceso al conocimiento es hoy mucho más estrecha de lo que era a mediados del siglo pasado.

María Ventocilla Cámara, luego de trabajar en la GUE Mercedes Cabello, fue directora de la Institución Esther Festini de Ramos Ocampo en el año 1964. De ella dice Rodolfo en su blog "Maruja -como le dicen- no tuvo una familia propia, no tuvo hijos y tal vez decidió no tenerlos porque pensaría que sería egoísta dedicarle su vida a unos pocos que ofrecerla a educar e instruir a muchos más en su profesión de educadora". Rita Castro Ramos fue Doctora en Pedagogía y Filosofía, docente de la Universidad Nacional Mayor de San Marcos, de la Universidad Inca Garcilaso de la Vega y de la Universidad San Martín de Porres, de la que fue, también, Rectora. Blanca Dávila Recio fundó un reconocido colegio en Lima.

En el campo de la Educación Especial se puede apreciar la obra valiosísima de Yolanda Liliana Mayo Ortega, fundadora y Directora General del Centro Ann Sullivan. En esta misma área se encuentra el trabajo de Nelly Villalobos Bustamante, exalumna de las Religiosas del Sagrado Corazón, fundadora del Instituto Santa Magdalena Sofía Barat.

La inclinación de Nelly hacia la educación nace de su interés por la infancia el cual se despierta desde pequeña al crecer en un hogar muy abierto al trato con los niños:

A mí me han gustado mucho los niños, creo que nos viene de familia, mi padre era dentista de niños, es decir, siempre hemos estado rodeados de muchos niños y muchos adolescentes, y eso es lo que despierta un poco el trato y el interés por cómo van desarrollándose en las diferentes etapas.

Este interés la llevó a desempeñarse como voluntaria en el Hospital del Niño y es, también, por este que su compañera de colegio, Grimanesa Wiese, la busca para proponerle presentarse a una beca para estudiar en México una carrera que en Perú no existía: una carrera para la atención a niños con severos problemas del lenguaje o sin habla. Luego de la evaluación, fue el Instituto Mexicano 
de Audición y Lenguaje el que becó a Nelly Villalobos y a otra persona más. Grimanesa Wiese otorgó, personalmente, dos becas y, así, fueron cuatro los que viajaron a prepararse $y$, al regresar, fundaron el Centro Peruano de Audición y Lenguaje (CPAL): Elvira Giró, Jorge Llona, la argentina María del Carmen Millé y Nelly Villalobos.

En México, Villalobos se graduó de profesora de sordos y de profesora terapista del lenguaje. Regresó al Perú con un título que en el país no existía y es que en la época de su retorno la Educación Especial casi no tenía presencia. Ella se orientó al trabajo con niños con problema específico del lenguaje comprensivo y expresivo, de origen heterogéneo, y fundó en un primer momento un consultorio que con el tiempo se convertiría en el Instituto Santa Magdalena Sofía Barat, el cual recibió el reconocimiento oficial para trabajar en los niveles de Educación Inicial y Educación Primaria en 1959; aunque, diez años después, obtuvo la autorización para ofrecer Educación Secundaria, nunca se dio esta atención, debido a que su meta era desarrollar las capacidades del niño durante la educación primaria para que pudiese incorporarse de manera óptima en la secundaria regular; esto mucho antes de que en el Perú se hablara de educación inclusiva.

Fue miembro, también, del equipo docente que inició la formación de profesoras especialistas en audición y lenguaje, gracias a un convenio firmado entre CPAL y la Pontifica Universidad Católica del Perú. Ha estado, asimismo, entre las fundadoras del Grupo Latinoamericano de Rehabilitación Profesional, el cual ha presidido en el Perú y del que ha sido miembro del equipo directivo internacional.

El centro fundado por Nelly Villalobos no es solo una institución que brinda servicios a menores con necesidades educativas especiales, sino, también, un centro de formación de profesionales especialistas: allí se forman profesoras, terapistas del lenguaje, terapistas ocupacionales y psicólogos, de diversas universidades, como la Universidad Femenina del Sagrado Corazón, la Universidad de Lima, la Pontificia Universidad Católica del
Perú, la Universidad Nacional Mayor de San Marcos y la Universidad Peruana Cayetano Heredia. Incluso, médicos residentes en psiquiatría infantil hacen pasantías en el Santa Magdalena.

No son pocas las exalumnas de las instituciones del Sagrado Corazón que han extendido por todo el país el impulso a la educación pública y privada y a la actividad cultural. El papel de las Religiosas del Sagrado Corazón está ligado a la educación de la mujer peruana desde 1876 año en que, a pedido del presidente Manuel Pardo, llegaron a Lima para hacerse cargo de la Escuela Normal de Preceptoras, la que tenía la misión «de formar preceptoras competentes que dirijan las escuelas que conforme a ley deben establecerse en las capitales de provincia de departamento», según lo dispuesto en el artículo 1 del Reglamento de la Escuela Normal de Mujeres, del 18 de marzo de 1878 (citado por Valladares, 2012). Fueron tres las religiosas que llegaron a Lima procedentes de Chile: la madre Enriqueta Purroy, la madre Henriette Fioretti y la hermana Mercedes Tapia.

Ligada a la vida de esta institución está la de la madre Socorro del Pilar Cardó Franco, quien conoció a la Congregación cuando era alumna del Instituto Pedagógico Nacional Monterrico (IPNM). En su Sullana natal, Pilar acompañaba a su madre, maestra de educación primaria y directora de una escuela marginal, a su centro de trabajo, y ese contacto con la educación sería lo que influiría en ella en el momento de elegir carrera. Por una prima hermana egresada de la Normal de Monterrico conocía la institución y, como se exigía en ese entonces, postuló al examen de selección por su departamento (Piura) lo que la trajo a Lima a postular e ingresar en la selección nacional.

Se graduó como maestra de educación secundaria, en la especialidad de Filosofía $y$ Literatura, $y$, desde entonces, se ha desempañado en prácticamente todos los roles que puede desempeñar una maestra: ha sido profesora de aula, directora de varios colegios de su congregación (Sophianum, de Trujillo, de Bagua, que ahora gestiona otra congregación) 
y Jefe de Departamento, Directora Académica y Directora General en Monterrico, en dos períodos, en la década del 90 y desde 2007 hasta ahora. Según señala, muchos de los docentes del Instituto han sido sus alumnos, y el conocerlos le facilita mucho su labor como directora.

La madre Pilar muestra una visión muy clara de lo que es la educación en el país: de la situación actual, de las limitaciones que hay que superar, de la exigencia que se debe dar a la formación docente.

Yo creo que los gobiernos se han preocupado mucho, mucho, por la matrícula de la educación, pero no de la calidad, $y$, en ese sentido, no siento que la educación haya sido prioritaria en las decisiones del gobierno; siempre ha quedado como en un segundo plano y la formación docente más aún.

Esto ha cambiado en los últimos 15 años; actualmente el Estado invierte más en la capacitación de los docentes y encuentra en el Instituto Pedagógico un aliado importante. Bajo la dirección de Pilar Cardó, el IPNM ha operativizado varios de los programas de capacitación que el Estado ofrece a los maestros.

Como directora de una de las instituciones formadoras de maestros más antiguas del Perú, es consciente que el atractivo de la profesión docente como opción de vida ha decaído mucho; son muy pocos los jóvenes que eligen la especialidad de Educación Secundaria y, seguramente por eso, muy pocas las instituciones de educación superior que la ofrecen; $y$, aunque educación inicial y educación primaria siguen siendo una opción para los jóvenes, su atractivo también va disminuyendo. Según ella, una de las causas de este declive es la campaña demoledora que se ha hecho contra la docencia menospreciando la profesión.

Otro aspecto doloroso de la educación peruana que le preocupa es la inequidad. $\mathrm{Si}$ bien durante muchas décadas el trabajo en la educación básica de la Congregación estuvo más ligado a la educación de las hijas de familias de clase económica alta, desde inicios de la década del 70, más específicamente, en el año 72, la Congregación hizo un viraje hacia el servicio de las comunidades marginadas, respondiendo así al pedido del Concilio Vaticano II. De los cuatro colegios que tenía la Congregación, solo uno era público: ese año entregó dos colegios al Estado (Chalet y Trujillo) y se quedó solo con el Sophianum como colegio privado. Aparte de eso, abrió obras de misión en zonas rurales, como Bambamarca, Bagua y Layo.

Para ella, una mujer que ha participado de forma trascendental en la formación de muchas generaciones de educadoras, el papel principal de la profesional de la educación es acompañar

Más que hacer clases magistrales es de acompañar los procesos de aprendizaje; estar cerca de los estudiantes, escucharles, dialogar, eso es muy importante porque la mayoría de estos jóvenes vive mucha soledad: los dos papás están trabajando, entonces, de alguna manera el docente tiene que cubrir ese vacío de sus alumnos.

Siguiendo la tarea que Santa Magdalena Sofía Barat encargaba a las hermanas, "Sean madres", la docente debe de traducir esa maternidad en cuidado, delicadeza, ternura, atención a las necesidades, cualidades que, según la hermana Pilar, son inherentes a la femineidad, sin querer decir con esto que los varones no sean capaces de manifestarlas.

La vida de Pilar Cardó es como la vida de su Congregación: mística y trabajo. Fiel a la misión encomendada por Dios y por el Perú, la Congregación fundó en Lima la Universidad Femenina del Sagrado Corazón, que inició su labor académica el 15 de abril de 1963. Recavarren y Lecaros (2003) afirman al respecto:

Las Religiosas del Sagrado Corazón, evaluaron la necesidad de ofrecer a las mujeres peruanas un espacio universitario que les permitiera llegar 
a ser profesionales con características propias; formación académica integral; seriedad científica en investigación; competencia profesional; vivencia y compromiso con los valores cristianos; y búsqueda de las soluciones a los más acuciantes problemas del Perú. Es decir, abrir un espacio para que la mujer peruana tuviera un campo donde alcanzar la excelencia en sus futuras profesiones. (p. 163)

Entre las mujeres gestoras de esta empresa destaca la entonces Vicaria de la Congregación, madre Carmen Cubero y la madre Graciela Marrou, su primera rectora.

\section{A MODO DE CONCLUSIÓN}

Se puede proyectar desde un inicio un área tanto en el ámbito político social como en la docencia, se encuentra mujeres representativas que han podido construir su espacio en la vida nacional peruana con trabajo continuo, desempeñando diversas funciones prácticamente sin detenerse en el trascurso de los años, recibiendo apoyo de su familia y de las personas cercanas. No obstante, si bien como colectivo la mujer profesional de la educación ha tenido una significativa presencia en la docencia, esto no se ha dado en la dirección política y en la producción de conocimiento científico.

Las cualidades particulares de la mujer para la docencia se han ido formando debido a una larga trayectoria cultural que la liga de manera particular con el cuidado y la educación de los niños. Sin embargo, esto no debe servir para relegarla a un desempeño invisibilizado en el ámbito escolar doméstico. La trayectoria de quienes, a pesar de las barreras, han logrado tener una participación trascendental en la vida nacional, debe impeler a trabajar por empoderar a la pedagoga, de tal manera que se acreciente el desarrollo de capacidades y contextos de participación para su desarrollo personal y el del Perú. 


\section{REFERENCIAS}

Consejo Nacional de Ciencia, Tecnología e Innovación Tecnológica. (2019). Registro de investigadores en ciencia y tecnología del sistema nacional de ciencia, tecnología e innovación tecnológica - SINACYT. Recuperado de http://regina.concytec.gob.pe/ReporteDina/ investigadorCalificado.zul

Chávez, T. (2006). La enseñanza de la historia del Perú en la educación secundaria durante la segunda mitad del siglo XX. Lima: PUCP - Fondo Editorial.

Ministerio de Educación (2019). Nómina de Ministros. Recuperado de http://www.minedu.gob.pe/ institucional/nomina.php

Morillo, E. (2002). Reformas educativas en el Perú del siglo XX. Recuperado de http://www.rieoei. org/deloslectores/233Morillo.PDF

Núñez, A. (2013, 21 de abril). Mercedes Cabanillas: "El Apra no es un conjunto de amigotes que se juntan para apostar en la política". La República. Recuperado de http://www.larepublica. pe/21-04-2013/mercedes-cabanillas-el-apra-no-es-un-conjunto-de-amigotes-que-se-juntan-paraapostar-en-la-politica

Recavarren, M. y Lecaros, F. (2003). Raíces y horizonte. Apuntes para una historia. Lima: Religiosas del Sagrado Corazón - Provincia del Perú.

Rodolfo. (30 de enero de 2011). El otoño de la amauta (Mensaje en un blog) Recuperado de http://rodolfoescribe.over-blog.es/article-el-otono-de-la-amauta-66137621.html

Sociedad de Investigación Educativa Peruana. (2019). Asociados y asociadas de la SIEP. Recuperado de http://www.siep.org.pe/quienes-somos/asociados-de-la-siep/

Valdés, T. (2000). De lo social a lo político. La acción de las mujeres latinoamericanas. Santiago de Chile: LOM.

Valladares, O. (2012). La incursión de las mujeres a los estudios universitarios en el Perú: 18751908. Cuadernos del Instituto Antonio de Nebrija, 15(1), 105-123. Recuperado de https://erevistas.uc3m.es/index.php/CIAN/article/view/1544/758

Fecha de recepción: 02-08-18

Fecha de aceptación: 04-11-18 